\title{
Uso de la harina de maní forrajero (Arachis pintoi Krapov \& WC Greg) en la alimentación de cuyes (Cavia porcellus L)
}

\author{
Use of pinto peanut (Arachis pintoi Krapov \& WC Greg) meal in guinea pig \\ feeding (Cavia porcellus $\mathrm{L}$ )
}

\author{
Alejandrina Sotelo M. ${ }^{1,6}$, Carlos Contreras M. ${ }^{1}$, Edgar Norabuena M. ${ }^{2}$, Gladys \\ Carrión C. ${ }^{1}$, Victoria Reátegui Q. ${ }^{3}$, Roxana Castañeda ${ }^{4,5}$
}

\section{Resumen}

\begin{abstract}
El objetivo del presente estudio fue evaluar el efecto del uso de harina de maní forrajero Arachis pintoi en cuyes en etapa de crecimiento. La duración experimental fue de 49 días, empleándose 64 cuyes machos destetados, dispuestos en un diseño de bloques completamente al azar con cuatro tratamientos $(0,5,10,15 \%$ de niveles de inclusión de harina de $A$. pintoi), con cuatro repeticiones y cuatro cuyes por repetición. Los resultados indican que no hubo diferencia significativa entre los niveles de $A$. pinto $i$ para ganancia de peso, consumo de alimento, conversión alimenticia, rendimiento de carcasa y retribución económica.
\end{abstract}

Palabras clave: Arachis pintoi; conversión alimenticia; cuyes; rendimiento de carcasa; retribución económica

\section{Abstract}

The aim of the present study was to evaluate the effect of pinto peanut Arachis pintoi in guinea pigs in the growing stage. The experiment lasted for 49 days and included 64 weaned male guinea pigs, arranged in a completely randomized block design with four treatments $(0,5,10,15 \%$ levels of $A$. pintoi meal), with four repetitions and four guinea pigs per repetition The results indicate that there was no significant difference between

\footnotetext{
${ }^{1}$ Facultad de Zootecnia, Universidad Nacional Agraria La Molina, Lima, Perú

${ }^{2}$ Facultad de Ingeniería Química y Textil, Universidad Nacional de Ingeniería, Lima, Perú

${ }^{3}$ Facultad de Agronomía, Universidad Nacional de la Amazonía Peruana, Iquitos, Perú

${ }^{4}$ Facultad de Ciencias Veterinarias y Biológicas, Universidad Científica del Sur, Lima, Perú

${ }^{5}$ Facultad de Ciencias Biológicas, Universidad Nacional Mayor de San Marcos, Lima, Perú

${ }^{6}$ E-mail: asotelo@lamolina.edu.pe
}

Recibido: 12 de diciembre de 2017

Aceptado para publicación: 19 de septiembre de 2018 
A. pintoi levels for body weight gain, feed intake, feed conversion, carcass yield and economic retribution.

Key words: Arachis pintoi; feed conversion; guinea pigs; carcass yield; economic retribution

\section{INTRODUCCIÓN}

Los forrajes son la base de la alimentación para los cuyes (Cavia porcellus). Dentro de las fabáceas tropicales se encuentra el maní forrajero (Arachis pintoi), especie de alto valor nutricional, de fácil acceso y manejo sencillo para el pequeño y mediano productor. Es una leguminosa de buena palatabilidad, tolerante al pastoreo intensivo y de condiciones de sombra, con altos rendimientos de forraje en zonas tropicales y permite mejorar los suelos y pastos degradados (Cab et al., 2008), además, es de fácil establecimiento bajo una amplia gama de condiciones climáticas y de suelo (Khamseekhiew et al., 2001). Tiene la capacidad de introducir nitrógeno dentro del sistema suelo-planta, se adapta a suelos ácidos con fertilidad media (Ferreira et al., 2012a) y tiene la habilidad para ser intercalado con diferentes pastos en la integración de los sistemas de cultivos y ganadería (Guimarães et al., 2016).

Según Bastiani y Pizarro (2005), esta especie sudamericana puede ser considerada tan importante como la alfalfa (Medicago sativa), la cual requiere corrección del suelo, altos niveles de fertilidad y manejo planificado, además de requerir prácticas agronómicas de conservación de forrajes para maximizar su potencial de producción y manejo controlado para evitar timpanismo en los animales que la consumen. El maní forrajero, a diferencia de la alfalfa, se adapta bien a altas humedades y temperaturas ambientales y tiene mínimos problemas con enfermedades o plagas.
Es importante resaltar, además, que las fabáceas son plantas ampliamente reconocidas por su importancia económica y cultural vinculada a la seguridad alimentaria, provisión de servicios y fuentes nutracéuticas (Castañeda et al., 2017). Debido a ello, la Asamblea General de las Naciones Unidas consideró denominar el año 2016 como el «Año Internacional de las Legumbres» (FAO, 2016).

Por otro lado, el III Censo Nacional Agropecuario realizado en el Perú en 1994 estimó que la selva aporta el 13\% del total de cuyes y $12.1 \%$ de las unidades agropecuarias (INEI, 1994). Los cuyes son criados para su venta en mercados y en forma ambulatoria, así como para el autoconsumo, fiestas patronales y ceremonias costumbristas. Es así que la cría de animales menores juega un papel primordial en la estrategia de vida de las familias asentadas en zonas productivamente marginales, donde los animales se encuentran íntimamente ligados a todas sus actividades y forman parte de su cosmovisión (Califano y Echazú, 2013; Castañeda et al., 2014). De esta manera, la selva del Perú constituye una importante plaza para la crianza de cuyes, representando una excelente oportunidad para la introducción de insumos nuevos como $A$. pintoi.

Arachis pintoi puede ser administrada como forraje fresco, heno o ensilado (Ferreira et al., 2012b). Andrade et al. (2016) sostienen que su uso es viable y sostenible en los diversos sistemas de producción animal del trópico. Malagón (2013), al evaluar diferentes niveles $(10,20$ y $30 \%)$ de harina de maní 
forrajero en la dieta de cuyes no encontró diferencias en la ganancia de peso, pero la inclusión de $20 \%$ obtuvo mayor beneficio económico. Asimismo, Alvares (2009) demostró que $A$. pintoi tuvo un efecto significativo sobre la conversión alimenticia en cuyes respecto a Axonopus scoparius y Malvaviscus arboreus, en tanto que Tuquinga (2015) determinó que $30 \%$ de inclusión de $A$. pintoi dio mejores rendimientos productivos desde el destete hasta el inicio de la vida reproductiva en conejos. No obstante, Alcívar (2012) y Oropesa et al. (2006) no encontraron diferencias estadísticas para los parámetros de peso, consumo de alimento y conversión alimenticia al evaluar varios niveles de harina de maní forrajero $(0,10,20,30 \%)$ en la alimentación de engorde de cuyes y conejos, respectivamente; así mismo, Nieves et al. (1997) tampoco encontraron diferencias en ganancias de peso en conejos suplementados con dietas que contenían $30 \%$ de maní forrajero frente a dietas control.

Otras investigaciones han demostrado los efectos benéficos de $A$. pintoi sobre el comportamiento productivo en animales de granja. Así, Andrade et al. (2015) en cerdos, Rojas (2007) y Andrade et al. (2014) en bovinos, Fernandes et al. (2013) y Schnaider et al. (2014) en ovinos. A. pintoi es pues una especie con potencial forrajero que puede ser reconocida como una buena alternativa en la alimentación animal frente a los cultivos tradicionales. La presente investigación se llevó a cabo con el fin de evaluar una fuente alimenticia que pueda ser incluida en el concentrado para cuyes y que contribuya a cubrir los requerimientos nutricionales y a un menor costo.

\section{MATERIALes y Métodos}

\section{Localización y Animales}

El experimento se realizó en las instalaciones del Programa de Investigación y Proyección Social en Carnes de la Facultad de
Zootecnia de la Universidad Nacional Agraria La Molina (UNALM), Lima, Perú.

Se utilizaron 64 cuyes machos mejorados de la línea Tipo 1 de $14 \pm 3$ días de edad, recién destetados, de la institución, con un peso inicial promedio de $280 \mathrm{~g}$. Se distribuyeron cuatro cuyes por poza, donde cada poza tenía un área de $0.5 \mathrm{~m}^{2}$ y conformaba una unidad experimental (UE). Las pozas eran de concreto armado y estaban revestidas de cemento. Como cama se utilizó una capa de $5 \mathrm{~cm}$ de coronta molida, con el fin de controlar la humedad. Cada tratamiento tuvo 4 UE, lo que hizo un total de 16 animales por dieta experimental. El peso vivo fue registrado semanalmente hasta culminar la etapa experimental de 49 días (correspondientes a la etapa de crecimiento). El control de peso de los animales y del concentrado se realizó utilizando una balanza de $3 \mathrm{~kg}$ de capacidad y con $2 \mathrm{~g}$ de aproximación.

\section{Alimento}

En las dos primeras semanas se suministró diariamente y ad libitum una dieta única, consistente solo de concentrado. Los comederos eran de arcilla enlozada, de forma ovalada y de $300 \mathrm{~g}$ de capacidad. Posteriormente y hasta el final del estudio se utilizaron comederos tolvas de plástico de $2 \mathrm{~kg}$ de capacidad. El agua fue suministraba diariamente y ad libitum en bebederos de arcilla enlozada, los cuales se cambiaban una vez al día.

Las dietas experimentales se formularon por programación lineal y al mínimo costo (Cuadro 1), obteniéndose raciones isoproteícas $(18 \%$ de proteína) e isoenergéticas ( $2.8 \mathrm{de} \mathrm{ED} \mathrm{Mcal} / \mathrm{kg}$ ), cubriendo las necesidades recomendadas por el NRC (1995) para cuyes en crecimiento. Los insumos de las dietas, incluyendo A. pintoi, fueron molidos utilizando un molino de seis cuchillas y una malla de $2 \mathrm{~mm}$ para la obtención de harina. Se tomó una muestra de cada una de las dietas y se realizó el análisis químico proximal según la metodología de la AOAC (2012). 
Cuadro 1. Composición porcentual y valor nutricional estimado de las dietas experimentales

\begin{tabular}{lcccc}
\hline & \multicolumn{4}{c}{ Niveles de harina de Arachis pintoi (\%) } \\
\cline { 2 - 5 } & 0 & 5 & 10 & 15 \\
\hline Afrecho de trigo & 61.38 & 67.03 & 65.92 & 64.81 \\
Harina de Arachis pintoi & 0.00 & 5.00 & 10.00 & 15.00 \\
Heno de alfalfa & 17.40 & 11.60 & 5.80 & 0.00 \\
Torta de soya & 12.28 & 10.78 & 11.08 & 11.37 \\
Maíz & 2.00 & 2.00 & 2.00 & 2.00 \\
Melaza de caña & 3.55 & 0.00 & 0.00 & 0.00 \\
Carbonato de calcio & 1.51 & 1.79 & 1.84 & 1.89 \\
Fosfato dicálcico & 0.36 & 0.11 & 0.17 & 0.23 \\
Sal común & 0.36 & 0.38 & 0.39 & 0.40 \\
Premezcla Min.-Vit. & 0.12 & 0.12 & 0.12 & 0.12 \\
Vitamina C & 0.06 & 0.06 & 0.06 & 0.06 \\
DL-Metionina & 0.18 & 0.17 & 0.17 & 0.18 \\
Cascarilla de arroz & 0.00 & 0.08 & 1.21 & 2.35 \\
Aceite de soya & 0.82 & 0.88 & 1.24 & 1.59 \\
\hline Total & 100.0 & 100.0 & 100.0 & 100.0 \\
\hline ED (Mcal/kg) & 2.80 & 2.80 & 2.80 & 2.80 \\
Proteína (\%) & 18.00 & 18.00 & 18.00 & 18.00 \\
Fibra (\%) & 10.00 & 10.00 & 10.00 & 10.00 \\
Calcio (\%) & 1.00 & 1.00 & 1.00 & 1.00 \\
Fósforo (\%) & 0.80 & 0.80 & 0.80 & 0.80 \\
Sodio (\%) & 0.20 & 0.20 & 0.20 & 0.20 \\
Lisina total (\%) & 0.85 & 0.84 & 0.84 & 0.85 \\
Metionina total (\%) & 0.43 & 0.43 & 0.43 & 0.43 \\
Met+Cist total (\%) & 0.74 & 0.74 & 0.74 & 0.74 \\
Arginina total (\%) & 1.21 & 1.22 & 1.22 & 1.22 \\
Treonina total (\%) & 0.65 & 0.65 & 0.65 & 0.66 \\
Triptófano total (\%) & 0.33 & 0.33 & 0.32 & 0.31 \\
Precio S.//kg & 0.92 & 0.90 & 0.91 & 0.92 \\
\hline
\end{tabular}

Se determinó la energía digestible y el valor nutricional del maní forrajero (Arachis pintoi), tanto de hojas como de tallos (Sotelo et al., 2016), presentado en forma de harina. La composición de aminoácidos de $A$. pintoi fue estimada mediante ecuaciones de predicción para la harina de alfalfa (NRC, 1994). El análisis químico proximal de la harina de A. pintoi se presenta en el Cuadro 2.

\section{Tratamientos}

Se evaluaron cuatro tratamientos con niveles de 0, 5, 10 y $15 \%$ de harina de Arachis pintoi, con sustitución progresiva del heno de alfalfa, de tal manera que en todos los tratamientos se obtuviera la misma cantidad de proteína (Cuadro 3). El objetivo fue evaluar el resultado de sustitución a igual nivel pro- 
Cuadro 2. Análisis químico proximal en base seca de la harina de Arachis pintoi

\begin{tabular}{lc}
\hline Nutrientes & Porcentaje \\
\hline Materia orgánica & 89.16 \\
Proteína cruda & 18.59 \\
Extracto etéreo & 1.55 \\
Fibra cruda & 23.18 \\
Extracto libre de N & 45.84 \\
Ceniza (\%) & 10.85 \\
\hline
\end{tabular}

Fuente: Laboratorio de Evaluación Nutricional de Alimentos del Departa-mento de Nutrición de la Facultad de Zootecnia (UNALM)

teico. El análisis proximal de las dietas experimentales se presenta en el Cuadro 4.

\section{Variables Evaluadas}

- Ganancia de peso. El peso vivo se determinó al inicio del experimento y en forma semanal. El peso fue registrado a la misma hora y con el animal en ayunas. La ganancia de peso se determinó por el peso vivo ganado semanalmente.

- Consumo de alimento. El consumo del concentrado y el control de peso se calcularon en las mismas fechas. El consumo fue medido por unidad experimental, para lo cual se restó el peso del alimento residuo al peso del alimento ofrecido. Los resul- tados se expresaron en gramos de materia seca.

- Conversión alimenticia. Se calculó considerando el consumo semanal dividido entre la ganancia de peso semanal.

- Rendimiento de carcasa. La carcasa incluye cabeza, patas y vísceras (hígado, corazón, pulmones, riñones y bazo). Se tomó un cuy al azar por cada unidad experimental (total: 16 cuyes), los mismos que fueron sometidos a ayuno por 24 horas antes del sacrificio. El rendimiento de la carcasa se calculó tomando en cuenta el peso vivo menos el peso de la carcasa y se expresó en porcentaje.

- Retribución económica. Se calculó mediante la diferencia de los ingresos (precio de la carne de cuy/kg por la ganancia de peso) y los egresos (costo del concentrado).

\section{Análisis Estadístico}

Se utilizó el diseño de bloques completamente al azar, con cuatro bloques (constituidos por rangos de peso de los cuyes al destete) y cuatro tratamientos (niveles de harina de $A$. pintoi), donde cada tratamiento estuvo formado por cuatro UE y cuatro cuyes por UE. Se realizó el análisis de variancia para comparar la variabilidad de las medias dentro de cada grupo con la variabilidad de toda la muestra (Dawson, 2009) y la prueba de Tukey (Calzada, 1982) para determinar la comparación de medias de los tratamientos de las variables evaluadas.

Cuadro 3. Niveles de sustitución de harina de Arachis pintoi por heno de alfalfa con similar aporte proteico para alimentación de cuyes

\begin{tabular}{cccc}
\hline Tratamientos & $\begin{array}{c}\text { Harina de } \text { A. pintoi } \\
(\%)\end{array}$ & $\begin{array}{c}\text { Heno de alfalfa } \\
(\%)\end{array}$ & $\begin{array}{c}\text { Aporte de proteína } \\
(\%)\end{array}$ \\
\hline T0 & 0 & 17.4 & 2.61 \\
T1 & 5.0 & 11.6 & 2.61 \\
T2 & 10.0 & 5.8 & 2.61 \\
T3 & 15.0 & 0 & 2.61 \\
\hline
\end{tabular}


Cuadro 4. Análisis químico proximal de las dietas experimentales

\begin{tabular}{lcccc}
\hline \multirow{2}{*}{ Nutrientes } & \multicolumn{4}{c}{ Niveles de harina de Arachis pintoi (\%) } \\
\cline { 2 - 5 } & 0 & 5 & 10 & 15 \\
\hline Humedad (\%) & 13.87 & 12.44 & 10.67 & 10.83 \\
Materia seca (\%) & 86.13 & 87.56 & 89.33 & 89.17 \\
Proteína total (\%) & 18.82 & 17.73 & 18.83 & 17.44 \\
Fibra cruda (\%) & 9.01 & 9.15 & 9.01 & 9.27 \\
Extracto etéreo (\%) & 1.23 & 2.94 & 4.12 & 4.35 \\
Extracto libre de N (\%) & 50.00 & 50.83 & 50.60 & 50.86 \\
\hline
\end{tabular}

Fuente: Laboratorio de Evaluación Nutricional de Alimentos del Departamento de Nutrición de la Facultad de Zootecnia (UNALM)

\section{Resultados y Discusión}

\section{Peso Vivo y Ganancia de Peso}

Los pesos promedios y las ganancias de peso por tratamiento se muestran en el Cuadro 5. Los cuyes del tratamiento T2 con $5 \%$ de $A$. pintoi tuvieron la mayor ganancia de peso $(723.0 \mathrm{~g})$ y mayor peso $(1002 \mathrm{~g})$ al final del experimento; sin embargo, las diferencias no llegaron a ser estadísticamente significativas. Al respecto, Alcívar (2012) y Malagón (2013) tampoco encontraron diferencias en las ganancias de peso a diferentes niveles de inclusión de $A$. pintoi en raciones para cuyes. Por otro lado, el peso final obtenido en todos los tratamientos se encuentra dentro del peso comercial de cuyes (>850 g) reportado por Morales et al. (2011) y similar a los registros obtenidos en la Granja de Cuyes de Cieneguilla de la UNALM (A. Sotelo, UNALM, comunicación personal). Estos resultados indican, en parte, que dietas con inclusión de $A$. pintoi tienen el mismo impacto nutricional en términos de rendimiento productivo en comparación con el uso de dietas que contiene insumos tradicionales como la alfalfa (tratamiento control, T0).

\section{Consumo de Alimento}

El consumo de alimento en términos de materia seca fue similar entre los cuatro tratamientos y bloques, sin diferencia significativa (Cuadro 6). Los niveles de harina de $A$. pintoi como reemplazo del heno de alfalfa en la dieta no afectaron la aceptabilidad por parte del animal (Church et al., 2003). Además, la fibra es de similar valor para todos los tratamientos en cuanto a retención, velocidad de flujo y densidad de fibra (Humphreys, 2005). Sin embargo, el consumo de alimento fue mayor en los estudios realizados por Mora (2015) y Alcivar (2012), lo cual probablemente se deba a la mayor inclusión de esta leguminosa en la dieta.

\section{Conversión Alimenticia}

Los resultados de conversiones alimenticias logradas en las siete semanas de evaluación tuvieron una tendencia similar a la ganancia de peso y al consumo de alimento. Los valores de conversión alimenticia fueron ligeramente superiores en los cuyes que recibieron la dieta con 5\% de A. pintoi (T1), seguidos de aquellos con la dieta de $15 \%$ de A. pintoi (T3). Sin embargo, las diferencias 
Cuadro 5. Ganancia de peso semanal (g) por cuy alimentado con dietas conteniendo cuatro niveles de Arachis pintoi

\begin{tabular}{|c|c|c|c|c|c|c|c|c|c|c|}
\hline & \multirow{2}{*}{$\begin{array}{l}\text { Peso } \\
\text { inicial }\end{array}$} & \multicolumn{7}{|c|}{ Semanas } & \multirow{2}{*}{$\begin{array}{l}\text { Peso } \\
\text { final }\end{array}$} & \multirow{2}{*}{$\begin{array}{l}\text { Gananci } \\
\text { de peso }\end{array}$} \\
\hline & & 1 & 2 & 3 & 4 & 5 & 6 & 7 & & \\
\hline T0 & 282.0 & 96.7 & 97.0 & 106.3 & 108.1 & 90.4 & 112.9 & 89.3 & 983 & $700.7^{\mathrm{a}}$ \\
\hline $\mathrm{T} 1$ & 279.0 & 120.6 & 93.0 & 98.9 & 95.9 & 111.6 & 98.0 & 105.1 & 1,002 & $723.0^{\mathrm{a}}$ \\
\hline $\mathrm{T} 2$ & 282.3 & 107.2 & 96.9 & 103.9 & 89.0 & 108.6 & 103.9 & 97.6 & 989 & $707.0^{\mathrm{a}}$ \\
\hline $\mathrm{T} 3$ & 283.5 & 106.5 & 87.5 & 109.7 & 98.2 & 109.4 & 104.9 & 101.0 & 1,001 & $717.1^{\mathrm{a}}$ \\
\hline
\end{tabular}

$\mathrm{TO}=$ dieta control; T1: con 5\% de A. pintoi; T2: con 10\% de A. pintoi; T3: con 15\% de A. pintoi

${ }^{a}$ Letras iguales dentro de una misma columna indican que no hay diferencia estadística $(p>0.05)$

Cuadro 6. Consumo semanal de alimento en materia seca $(\mathrm{g})$ en cuyes alimentados con dietas conteniendo Arachis pintoi a diferentes niveles

\begin{tabular}{ccccccccc}
\hline \multirow{2}{*}{ Tratamiento } & \multicolumn{7}{c}{ Semanas } & $\begin{array}{c}\text { Consumo } \\
\text { total }\end{array}$ \\
\cline { 2 - 8 } & 1 & 2 & 3 & 4 & 5 & 6 & 7 & $2702.7^{\mathrm{a}}$ \\
\hline T0 & 220.2 & 305.8 & 355.6 & 397.7 & 437.0 & 483.6 & 502.9 & $262.8^{\mathrm{a}}$ \\
T1 & 231.9 & 309.3 & 353.8 & 377.8 & 407.4 & 452.7 & 495.9 & 262.6 \\
T2 & 233.0 & 320.6 & 376.0 & 379.4 & 410.6 & 472.4 & 515.6 & $2707.7^{\mathrm{a}}$ \\
T3 & 225.8 & 306.5 & 387.7 & 382.3 & 426.6 & 457.1 & 520.3 & $2706.3^{\mathrm{a}}$ \\
\hline
\end{tabular}

T0 = dieta control; T1: con 5\% de A. pintoi; T2: con 10\% de A. pintoi; T3: con 15\% de A. pintoi

a Letras iguales dentro de una misma columna indican que no hay diferencia estadística $(p>0.05)$

Cuadro 7. Conversión alimenticia en cuyes alimentados con dietas conteniendo Arachis pinto $i$ a diferentes niveles

\begin{tabular}{ccccccccc}
\hline \multirow{2}{*}{ Tratamiento } & \multicolumn{7}{c}{ Semanas } & $\begin{array}{c}\text { Conversiór } \\
\text { alimenticic }\end{array}$ \\
\cline { 2 - 9 } & 1 & 2 & 3 & 4 & 5 & 6 & 7 & $3.89^{\mathrm{a}}$ \\
\hline T0 & 2.3 & 3.2 & 3.4 & 3.7 & 4.8 & 4.3 & 5.6 & $3.67^{\mathrm{a}}$ \\
T1 & 1.9 & 3.3 & 3.6 & 3.9 & 4.0 & 4.2 & 4.7 & $3.85^{\mathrm{a}}$ \\
T2 & 2.2 & 3.3 & 3.6 & 4.3 & 3.8 & 4.6 & 5.3 & $3.78^{\mathrm{a}}$ \\
T3 & 2.1 & 3.5 & 3.5 & 3.9 & 3.9 & 4.4 & 5.2 & \\
\hline
\end{tabular}

T0 = dieta control; $\mathrm{T} 1$ : con $5 \%$ de A. pintoi; T2: con 10\% de A. pintoi; T3: con 15\% de A. pintoi

${ }^{a}$ Letras iguales dentro de una misma columna indican que no hay diferencia estadística $(p>0.05)$ 
Cuadro 8. Rendimiento de carcasa (\%) en cuyes alimentados con dietas conteniendo Arachis pintoi a diferentes niveles

\begin{tabular}{cccccc}
\hline Tratamientos & $\begin{array}{c}\text { Peso vivo } \\
(\mathrm{g})\end{array}$ & $\begin{array}{c}\text { Merma } \\
(\mathrm{g})\end{array}$ & $\begin{array}{c}\text { Peso de } \\
\text { carcasa }(\mathrm{g})\end{array}$ & $\begin{array}{c}\text { Rendimiento } \\
\text { de carcasa } \\
(\%)\end{array}$ & $\begin{array}{c}\text { Retribución } \\
\text { económica } \\
(\mathrm{S} / .)\end{array}$ \\
\hline T0 & 953.5 & 242.0 & 711.5 & 74.6 & 16.76 \\
T1 & 983.5 & 256.5 & 727.0 & 74.0 & 17.34 \\
T2 & 947.0 & 254.0 & 693.0 & 73.2 & 17.03 \\
T3 & 991.5 & 247.5 & 744.0 & 74.9 & 17.22 \\
\hline
\end{tabular}

T0 = dieta control; T1: con 5\% de A. pintoi; T2: con 10\% de A. pintoi; T3: con 15\% de A. pintoi

observadas no llegaron a ser estadísticamente diferentes (Cuadro 7), lo que indica que $A$. pintoi tiene el mismo impacto nutricional que la alfalfa. Este resultado concuerda con lo hallado por Alcívar (2012), quién utilizando inclusiones de $0,10,20$ y $30 \%$ de esta harina en raciones de engorde de cuyes obtuvo conversiones de $2.96,2.91,3.10$ y 3.21 , respectivamente. Malagón (2013) también obtuvo conversiones alimenticias de 3.73, 3.99 y 4.38 para inclusiones de 10,20 y $30 \%$ de $A$. pintoi y Tripsacum laxum (pasto guatemala) en raciones para cuyes, no existiendo diferencias estadísticas.

\section{Rendimiento de Carcasa}

El rendimiento de la carcasa fue estadísticamente similar para los cuatro tratamientos (Cuadro 8). Estos resultados fueron similares a los obtenidos por Carbajal (2015), quien obtuvo rendimientos de carcasa entre 72.4 y $75.1 \%$, pero mayores al $65 \%$ reportado por Xicohtencatl-Sánchez et al. (2013).

\section{Retribución Económica del Alimento}

El balance de la retribución económica no arrojó diferencias significativas al emplear los tres niveles de harina de $A$. pintoi del estudio, concordando con lo determinado por Malagón (2013).

\section{Conclusiones}

Los niveles de inclusión en las dietas de la harina de Arachis pintoi de 0, 5, 10 y 15\% en reemplazo del heno de alfalfa no mostraron diferencias estadísticas en el comportamiento productivo en cuyes destetados.

\section{Literatura Citada}

\section{1. [AOAC] Association of Official} Analytical Chemist. Official methods of analysis of the Association of Official Analytical Chemist. Washington DC: AOAC.

2. Alvares L. 2009. Determinación del consumo voluntario de materia seca en tres forrajes Axonopus scoparius, Arachis pintoi, Malvaviscus arboreus y su influencia en el incremento diario de peso en cuyes hembras en crecimiento. Tesis de Ing. Agropecuario. Ecuador: Univ. Estatal Amazónica. 55 p.

3. Alcívar J Fabricio. 2012. Utilización de harina de maní forrajero (Arachis pintoi) en la alimentación de cobayos (Cavia porcellus) en la Parroquia La Unión, Provincia de los Ríos. Tesis de Médico Veterinario Zootecnista. Ecuador: Univ. Técnica de Cotopaxi. 108 p. 
4. Andrade EA, Ribeiro HMN, Liz DM, Almeida JGR, Miguel MF, Raupp GT, Ramos FR, Almeida EX. 2014. Herbage intake, methane emissions and animal performance of steers grazing dwarf elephant grass with or without access to Arachis pintoi pastures. Trop Grasslands 2: 4-5.

5. Andrade V, Lima R, Vargas J. 2015. Evaluación de la digestibilidad in vivo y concentración energética de dietas con harina de forraje de Arachis pintoi para cerdos en crecimiento y ceba. Rev Comp Prod Porcina 22: 209-212.

6. Andrade V, Lima $R$, Vargas J, Vargas S. 2016. Situación actual y perspectiva del multiuso de Arachis pintoi en agroecosistemas dedicados a la producción animal. Ctro Agríc 43: 80-87.

7. Bastiani N, Pizarro EA. 2005. Potencial forrajero del género Arachis en el trópico americano. En: IX Seminario de Pastos y Forrajes. [Internet]. Disponible en: http://www.avpa.ula.ve/eventos/ ix_seminario_pastosyforraje/Conferencias/C2-NaylosBastiani.pdf

8. Cab FE, Enríquez JF, Pérez J, Hernández A, Herrera JG, Ortega E, Quero AR. 2008. Potencial productivo de tres especies de Brachiaria en monocultivo y asociadas con Arachis pintoi en Isla, Veracruz. Tec Pecu Méx 46: 317-332.

9. Califano M, Echazú F. 2013. Etnobotánica en comunidades pastoriles. Conocimiento tradicional sobre especies tóxicas para el ganado en la cuenca del río Iruya (Salta, Argentina) Bol Soc Argent Bot 48: 253-265. doi: 10.31055/ 1851.2372.v48.n2.6271

10. Calzada BJ. 1982. Métodos estadísticos para la investigación. $2^{\text {a }}$ ed. Lima: Jurídica. $644 \mathrm{p}$.

11. Carbajal C. 2015. Evaluación preliminar de tres alimentos balanceados para cuyes (Cavia porcellus) en acabado en el Valle del Mantaro. Tesis de Ing. Zootecnista. Lima: Univ. Nacional Agraria la Molina. $70 \mathrm{p}$.
12. Castañeda R, Albán J, Gutiérrez H, Cochachin E, La Torre MI. 2014. Plantas silvestres empleadas como alimento para animales en Pisha, Ancash. Ecol Apl 13: 153-168.

13. Castañeda R, Gutiérrez H, Carrillo E, Sotelo A. 2017. Leguminosas (Fabaceae) silvestres de uso medicinal del distrito de Lircay, provincia de Angaraes (Huancavelica, Perú). Bol Latinoam Caribe Plant Med Aromat 16: 136-149.

14. Church DC, Pond WG, Pond KR. 2003. Fundamentos en nutrición y alimentación de animales. $2^{\circ}$ ed. México DF: Grupo Noriega Ed. 635 p.

15. Dawson GF. 2009. Interpretación fácil de la bioestadística: la conexión entre la evidencia y las decisiones médicas. Barcelona: Elsevier. $190 \mathrm{p}$.

16. Fernandes GM, Possenti RA, Teizeira de Mattos WT, Schammass EA, Ferrari E. 2013. In situ degradability and selected ruminal constituents of sheep fed with peanut forage hay. Arch Anim Nutr 67: 393-405. doi: 10.1080/ 1745039X.2013.834581

17. Ferreira AL, Mauricio RM, Pereira LGR, Azevedo JAG, Oliveira LS, Pereira JM. 2012a. Nutritional divergence in genotypes of forage peanut. Rev Bras Zootec 41: 856-863. doi: 10.1590/S1516-35982012000400005

18. Ferreira AL, Mauricio RM, Fernandes FD, Carvalho MA, Ramos AKB, Guimaraes R. 2012b. Ranking contrasting genotypes of forage peanut based on nutritive value and fermentation kinetics. Anim Feed Sci Technol 175: 1623. doi: 10.1016/j.anifeedsci.2012.03.024

19. [FAOJ Organización de las Naciones Unidas para la Agricultura y la Alimentación. 2016. Año internacional de las legumbres. A/RES/68/231. [Internet]. Disponible en: http://www.fao.org/pulses-2016/es/

20. Guimarães W, Aparecida de Pinho K, Soares P, Castro R, Alves D, Tavares L. 2016. Silage quality of corn and 
sorghum added with forage peanuts. Rev Caatinga 29: 465-472. doi: 10.1590/198321252016v29n224rc

21. [INEI] Instituto Nacional de Estadística e Informática. 1994. Estructura y dinámicas del espacio agropecuario, Perú en mapas: III Censo Nacional Agropecuario 1994. Lima. Perú. [Internet]. Disponible en: http://horizon.documentation.ird.fr/exl-doc/pleins_textes/doc3407/010016424.pdf

22. Humphreys LR. 2005. Tropical pasture utilization. New York: Cambridge University Press. 206 p.

23. Khamseekhiew B, Liang JB, Wong $C C$, Jalan $Z A$. 2001. Ruminal and intestinal digestibility of some tropical legume forages. Asian-Aust J Anim Sci 14: 321-325. doi: 10.5713/ajas.2001.321

24. Malagón M. 2013. Pasto guatemala (Tripsacum Laxum) con maní forrajero (Arachis pintoi) en la alimentación de cuyes (Cavia porcellus Linnaeus) en la etapa de engorde en la Maná-Cotopaxi. Tesis de Grado. Ecuador: Universidad Técnica Estatal de Quevedo. 63 p.

25. Mora M. 2015. Utilización de mezclas forrajeras de clima trópico húmedo para la alimentación de cuyes en la etapa de crecimiento-engorde. Tesis de Ing. Zootecnista. Riobamba, Ecuador: Escuela Superior Politécnica de Chimborazo. $95 \mathrm{p}$.

26. Morales A, Carcelén F, Ara M, Arbaiza T, Chauca L 2011. Evaluación de dos niveles de energía en el comportamiento productivo de cuyes (Cavia porcellus) de la raza Perú. Rev Inv Vet 22: 177-182. doi: 10.15381/rivep.v22i3.254

27. [NRC] National Research Council. 1994. Nutrient requirements of poultry. $9^{\text {th }}$ ed. Washington DC: National Academy Press. 155 p.
28. [NRC] National Research Council. 1995. Nutrient requirements of laboratory animals. Guinea pig. Washington DC: National Academy Press. 176 p.

29. Nieves D, Santana L y Benvent J. 1997. Niveles crecientes de Arachis pintoi (Krap y Greg.) en dietas en forma de harina para conejos de engorde. Arch Latinoam Prod Anim 5: 321-323.

30. Oropeza M, Terán O, Nieves D. 2006. Arachis pintoi en dietas para conejos de engorde. Rev Unell Cienc Tec 24: 87-92.

31. Rojas A. 2007. Ventajas y limitaciones para el uso del maní forrajero perenne (Arachis pintoi) en la ganadería tropical. En: XI Seminario Manejo y Utilización de Pastos y Forrajes en Sistemas de Producción Animal. Barquisimeto. Venezuela.

32. Schnaider MA, Ribeiro HMN, Kozloski GV, Reiter T, Orsoletta, ACD, Dallabrida $\boldsymbol{A L}$. 2014. Intake and digestion of wethers fed with dwarf elephant grass hay with or without the inclusion of peanut hay. Trop Anim Health Prod 46: 975-980. doi: 10.1007/ s11250-014-0594-5

33. Sotelo A, Contreras C, Norabuena E, Castañeda $R$, van Heurck M, Bullón $C$. 2016. Digestibilidad y energía digestible de cinco leguminosas forrajeras tropicales. Rev Soc Quím Perú 82: 306-214.

34. Tuquinga J. 2015. Evaluación de harina de Arachis pintoi y su efecto en la alimentación de conejos desde el destete hasta el inicio de la vida reproductiva. Tesis para obtener el título de Ingeniero Zootecnista. Ecuador: Escuela Superior Politécnica de Chimborazo. $99 \mathrm{p}$.

35. Xicohtencatl-Sánchez PG, BarreraZúñiga S, Orozco-Orozco T, TorresSandoval F, Monsiváis- Isiordia $R$. 2013. Parámetros productivos de cuyes (Cavia porcellus) del nacimiento al sacrificio en Nayarit, México. Abanico Vet 3: 36-43. 